\title{
New insights into the properties of high-manganese steel
}

\author{
Wolfgang Bleck \\ Steel Institute, RWTH Aachen University, Germany \\ (Received: 5 June 2020; revised: 10 August 2020; accepted: 12 August 2020)
}

\begin{abstract}
In the Collaborative Research Centre 761's "Steel ab initio - quantum mechanics guided design of new Fe based materials," scientists and engineers from RWTH Aachen University and the Max Planck Institute for Iron Research conducted research on mechanism-controlled material development with a particular focus on high-manganese alloyed steels. From 2007 to 2019, a total of 55 partial projects and four transfer projects with industrial participation (some running until 2021) have studied material and process design as well as material characterization. The basic idea of the Collaborative Research Centre was to develop a new methodological approach to the design of structural materials. This paper focuses on selected results with respect to the mechanical properties of high-manganese steels, their underlying physical phenomena, and the specific characterization and modeling tools used for this new class of materials. These steels have microstructures that require characterization by the use of modern methods at the nm-scale. Along the process routes, the generation of segregations must be taken into account. Finally, the mechanical properties show a characteristic temperature dependence and peculiarities in their fracture behavior. The mechanical properties and especially bake hardening are affected by short-range ordering phenomena. The strain hardening can be adjusted in a never-before-possible range, which makes these steels attractive for demanding sheet-steel applications.
\end{abstract}

Keywords: high-manganese steels; stacking fault energy; twinning; short-range ordering; mechanical properties

\section{Introduction}

Since the mid-1990s, considerable progress had been achieved in modeling and measurement technologies, which has enabled the systematic knowledge-based development of the particularly demanding new material group of high-manganese alloyed steels. With these high-manganese steels (HMnS; up to $30 \mathrm{wt} \% \mathrm{Mn}$ ), extraordinary phenomena in plastic deformation are observed and, on this basis, exceptional property combinations can be realized. As a central scientific working hypothesis of the Collaborative Research Centre 761 (CRC), which was established by Deutsche Forschungsgemeinschaft DFG in 2007, it was assumed that the stacking fault energy (SFE), in particular, controls the deformation mechanisms. The SFE thus served as a parameter for the development of processes and materials, as it is experimentally accessible and can also be calculated based on thermodynamic models and with modern ab-initio methods.

In recent years, HMnS have become an innovative research focus in many places around the world. The investigations range from fundamental questions about the deformation mechanisms to questions of manufacturability, to technological investigations into the application potential with a special focus on applications in automotive engineering and security technology. The first review articles on this steel group include Refs. [1-4]. This broad spectrum of topics was also evident at the "High Manganese Steels 2019" conference organized by the CRC in Aachen, in which 200 scientists participated [5-6]. Larger consortia on the subject of $\mathrm{HMnS}$ can be found in Korea, particularly at the universities POSTECH, Yonsai, and Seoul National, in China coordinated mainly by the Central Iron and Steel Research Institute (CISRI), in the USA with a focus on medium-manganese steels (MMnS) at the Colorado School of Mines, and in Europe at the universities in Leuven, Metz, and Paris.

More recently, application-related research approaches have focused on somewhat lower manganese contents and multiphase structures, which are often referred to as MMnS or advanced high-strength steels (AHSS) 3rd generation. The aim of this research is to apply the phenomena of the mechanically induced transformations of metastable austenite observed in HMnS to multiphase structures. The lower manganese contents make these steels easier to manufacture in the steel shop, as well as during down-stream processing, while also reducing the alloying costs and providing attractive properties, especially for automotive sheet applications. 
The variety of deformation mechanisms observed in fully austenitic HMnS (SLIP: dislocation slip; TRIP: transformation-induced plasticity; TWIP: twinning-induced plasticity; MBIP: microband-induced plasticity) requires a basic understanding of the occurrence of the various deformation modes and the resulting material properties. Therefore, in this paper, the most important parameters influencing the material behavior, including alloy composition, microstructure, and test temperature, were examined. For all the steel concepts, the microstructure required quantitative description at the nmscale in order to understand the various plasticity phenomena and to develop structure-property correlations. Furthermore, the process paths also required adaptation to the high-alloy content to avoid segregations and to generate the desired microstructure.

\section{Materials}

A total of more than 30 materials were examined at the CRC. Table 1 lists the chemical compositions of those materials relevant to the results reported in this paper together with the calculated SFEs.

Table 1. Chemical compositions of the materials examined at the Collaborative Research Centre and the calculated SFEs

\begin{tabular}{lccccccc}
\hline Steel grade & $\mathrm{C} / \mathrm{wt} \%$ & $\mathrm{Mn} / \mathrm{wt} \%$ & $\mathrm{Al} / \mathrm{wt} \%$ & $\mathrm{Si} / \mathrm{wt} \%$ & $\mathrm{Cr} / \mathrm{wt} \%$ & $\mathrm{~N} / \mathrm{ppm}$ & $\mathrm{SFE} /\left(\mathrm{mJ} \cdot \mathrm{m}^{-2}\right)$ \\
\hline X30Mn22 & 0.31 & 22.28 & - & 0.12 & 0.24 & 170 & 17 \\
X60Mn18 & 0.53 & 17.88 & - & 0.08 & - & 280 & 17 \\
X60Mn22 & 0.54 & 22.26 & - & 0.21 & - & 120 & 23 \\
X40MnCrAl19-2-1 & 0.39 & 18.90 & 1.19 & 0.31 & 1.70 & 121 & 26 \\
X60MnAl17-1 & 0.60 & 16.85 & 1.14 & 0.06 & 0.26 & 80 & 25 \\
X120MnAl30-8 & 1.11 & 29.80 & 7.65 & 0.05 & 0.03 & 80 & 25 \\
X20Mn5 & 0.19 & 4.02 & 0.03 & 0.50 & - & 110 & n.d. \\
DP800 & 0.13 & 1.51 & 0.04 & 0.23 & - & 18 & n.d. \\
TRIP700 & 0.19 & 1.75 & 1.00 & 0.35 & - & 20 & n.d. \\
\hline
\end{tabular}

Note: The industrially produced DP800 and TRIP700 steels serve as reference materials.

Two main effects of alloying on the physical metallurgy of HMnS are observed: (1) stabilization of austenite and/or formation of a single-phase austenitic microstructure, and (2) adjustment of the SFE for the sake of the activation or suppression of different deformation mechanisms.

While manganese, carbon, and aluminum can efficiently affect the SFE, their roles in the stabilization or destabilization of austenite differ. In fact, both manganese and carbon cause the system to develop a fully austenitic microstructure, whereas aluminum is a ferrite-forming element. Particular attention was paid to the transition of the deformation mechanisms from TWIP to TRIP for Fe-Mn-C alloys with an SFE of approximately $20 \mathrm{~mJ} / \mathrm{m}^{2}$. A manganese content between $17 \mathrm{wt} \%$ and $30 \mathrm{wt} \%$ was selected to adjust the SFE and enable investigation of the predominant deformation mechanisms. A maximum carbon content of $0.6 \mathrm{wt} \%$ was selected to avoid carbide precipitation.

Later, the alloy system was expanded to include high contents of up to $11 \mathrm{wt} \%$ of the element aluminum. On one hand, this allowed the SFE and thus the formation of twins to be influenced; on the other hand, a high-aluminum content also made it possible to investigate the MBIP phenomenon and the effect of the aluminum-rich $\kappa$-phase on the deformation behavior. The investigated highly alloyed $\mathrm{Fe}-\mathrm{Mn}-\mathrm{Al}-\mathrm{C}$ steels with manganese contents of more than $25 \mathrm{wt} \%$, aluminum contents of more than $8 \mathrm{wt} \%$, and carbon contents of more than $0.8 \mathrm{wt} \%$ have been of special interest because of their high SFEs, which can reach levels greater than $80 \mathrm{~mJ} / \mathrm{m}^{2}$. Furthermore, these steels show the formation of a nano-sized $\kappa$-phase and $\kappa$-carbides with a perovskite structure and the approximate composition of $(\mathrm{Fe}, \mathrm{Mn})_{3} \mathrm{AlC}$. A significant planar glide on a single slip plane is observed during plastic deformation. When small strains are applied, the rearrangement of dislocations that lack three-dimensional (3D) mobility results in the formation of a finely organized structure. In addition, crystallographic microbands and microband intersections occur at medium to high strains. The geometrically necessary microbands result in a continuous strain hardening, which provides an excellent combination of strength and formability.

Finally, the range of materials was expanded to include steels with lower manganese contents (e.g., $<12 \mathrm{wt} \%$ rather than $>17 \mathrm{wt} \%$ ). After suitable heat treatment, these MMnSs have a two-phase structure comprising metastable austenite and martensite. Because of their processing, MMnSs are prone to partitioning effects of the alloying elements, especially carbon but to some lesser extent also manganese and other substitutional elements, which results in pronounced differences in local chemical compositions. Many of these steels are characterized by bimodal grain-size distributions. The scientific challenge is evident in the fact that the various deformation mechanisms of austenite continue to work, but the austenitic phase is integrated into a cubic, body-centered matrix. The mechanical, crystallographic, and chemical in- 
teractions at the interfaces therefore play an important role.

The relatively high $\mathrm{N}$ contents of the laboratory steels are due to the high $\mathrm{N}$ affinity of Mn-containing melts. The industrially produced DP800 and TRIP700 steels served as reference materials and provided benchmarks for the mechanical properties.

In the beginning, the SFE was considered to be the primary control variable, but a more detailed structural description took into account grain size and segregation effects as well as the volume fractions, sizes, and morphologies of the components as important parameters. The dependency of deformation twinning on grain size was found to result in significant variations in strain-hardening behavior, strain-rate sensitivity, and serrated flow in the investigated Fe-Mn-C TWIP steels. The mechanical properties of steels examined naturally depend heavily on the respective manufacturing conditions. Table 2 provides an overview of the mechanical properties obtained in the laboratory in the fully annealed condition for 1-mm-thick cold-rolled sheets. The microstructure describes the annealed condition prior to mechanical testing. It is noted that the properties of X120MnAl30-8 steel are determined by the $\kappa$-phase precipitation and those of $\mathrm{X} 20 \mathrm{Mn} 5$ steel by the fractions of the different constituents, both of which are very dependent on the selected annealing conditions, so that the values given in Table 2 can serve only as a guide.

Table 2. Tensile test properties and microstructures in the fully annealed condition for 1-mm-thick cold-rolled sheet

\begin{tabular}{|c|c|c|c|c|c|}
\hline Steel grade & $\mathrm{YP} / \mathrm{MPa}$ & UTS / MPa & $\mathrm{UE} / \%$ & $\mathrm{TE} / \%$ & Microstructure \\
\hline $\mathrm{X} 30 \mathrm{Mn} 22$ & 380 & 820 & 45 & 49 & $\gamma$ \\
\hline $\mathrm{X} 60 \mathrm{Mn} 18$ & 350 & 980 & 56 & 57 & $\gamma$ \\
\hline $\mathrm{X} 60 \mathrm{Mn} 22$ & 460 & 1080 & 49 & 50 & $\gamma$ \\
\hline $\mathrm{X} 40 \mathrm{MnCrAl19-2-1}$ & 530 & 940 & 45 & 48 & $\gamma$ \\
\hline X60MnA117-1 & 465 & 980 & 57 & 66 & $\gamma$ \\
\hline X120MnAl30-8 & 610 & 900 & 40 & 42 & $\gamma+\kappa$ \\
\hline $\mathrm{X} 20 \mathrm{Mn} 5$ & 740 & 1190 & 21 & 26 & $\gamma+\alpha^{\prime}$ \\
\hline DB800 & 350 & 800 & 13 & 17 & $\alpha+\alpha^{\prime}$ \\
\hline TRIP700 & 470 & 710 & 23 & 30 & $\alpha+\alpha_{\mathrm{B}}+\gamma_{\mathrm{r}}$ \\
\hline
\end{tabular}

Note: YP-Yield point; UTS-Ultimate tensile strength; UE-Uniform elongation; TE-Total elongation of specimens with 80-mm gauge length.

Two laboratory process routes were investigated in the manufacture of hot- and cold-rolled sheet materials, as shown in Fig. 1: (1) ingot casting with cross-section dimensions of $160 \mathrm{~mm} \times 160 \mathrm{~mm}$, open die forging, homogenization annealing, and hot rolling to a final thickness of $2 \mathrm{~mm}$, maximum width of $200 \mathrm{~mm}$; (2) two-roll strip casting with a cast thickness between 1.5 and $3.0 \mathrm{~mm}$, a width of $145 \mathrm{~mm}$, and strip lengths between 45 and $100 \mathrm{~m}$, partially inline hot rolling of $25 \%$, and homogenization annealing.

Each of these process routes was intensively examined and then compared with regard to the resulting strip properties. Compared to the conventional process route, strip casting enabled the production and further processing of TRIP/TWIP/MBIP steels with very high manganese and aluminum contents on a greatly shortened process route. The strip-casting structure is characterized by two directionally
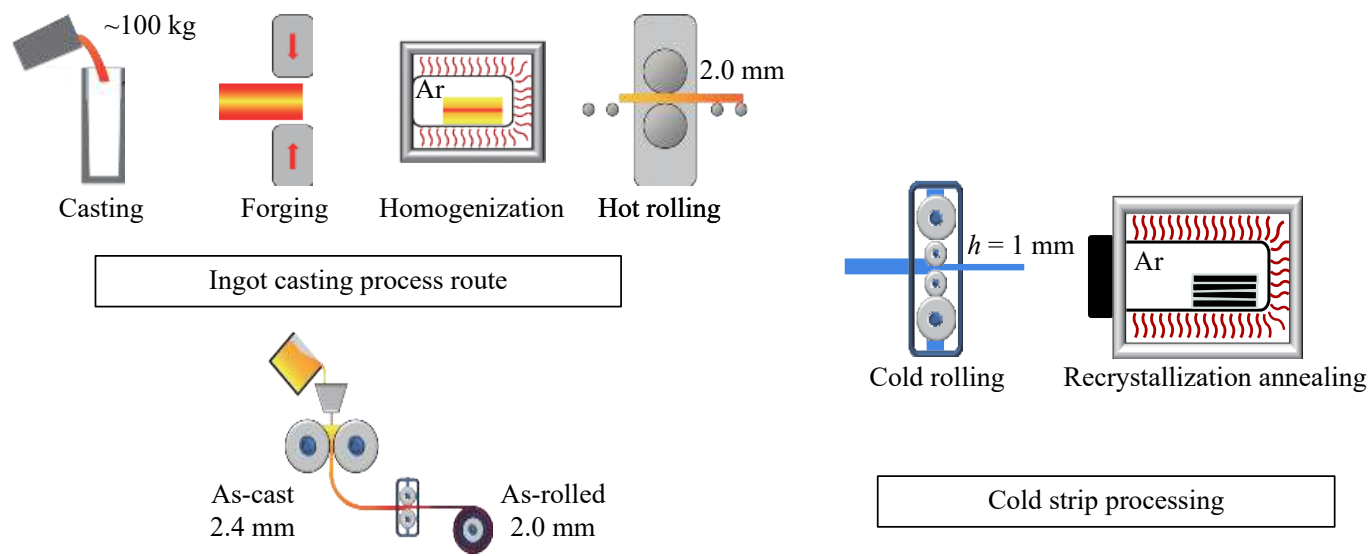

Strip casting process route

Cold strip processing

Fig. 1. Laboratory process routes for hot- and cold-rolled sheet samples. 
solidified band shells, which are assembled in the casting gap. The fine solidification structure was characterized by a secondary dendrite arm spacing range of 3-10 $\mu \mathrm{m}$. The micro segregations measured by electron probe micro analysis (EPMA) were comparable to ingot casting, but had very short wavelengths, which could be resolved much more quickly by subsequent annealing treatment. With the homogenization heat treatment, the manganese segregation amplitudes could be reduced from $\sim 4 \mathrm{wt} \%$ after ingot casting to less than $1 \mathrm{wt} \%$. The line scans showed manganese and carbon to have segregation behavior opposite to that of aluminum. A high level of purity was observed along with very small nonmetallic inclusions $(\sim 1 \mu \mathrm{m})$ in relative large numbers. Compounds of $\mathrm{Si}, \mathrm{Al}, \mathrm{S}, \mathrm{Se}, \mathrm{O}$, and $\mathrm{N}$ were found. An inline rolling degree of $25 \%$ was necessary to close all central pores and to improve the surface quality. The maximum cast strip thickness that could be produced was increased to $3.0 \mathrm{~mm}$ in the course of the project. Nevertheless, to some extent this limited the development of hot-rolling or hot-plus-coldrolling schedules in the development of fine austenitic grain structures. Furthermore, the ingot-casting process route was combined with a vacuum melting furnace to enable more precise metallurgical preparation, especially of high-Al-containing steels. The materials were investigated in hot- or coldrolled and annealed states. In this paper, most results refer to 1-mm-thick cold-rolled material.

In many of the steels currently produced, the primary microstructure formed during solidification is of minor importance because phase transformations and recrystallizations yield a secondary microstructure that is essential to the mechanical properties. However, the primary structure is of great- er interest in the case of high-alloy steels, and in particular high-alloy austenitic steels that do not undergo phase transformation during production. Parameters that influence the segregation are critical and must be controlled. Furthermore, macro- and microscale fluid flow as well as solid-state diffusion must be taken into account to fully understand the local chemical composition that affects the microstructural development and ultimately the mechanical properties [7]. In general, single-phase austenitic solidifying materials are observed to exhibit significantly lower fluctuations in concentration than ferritic-austenitic solidifying grades.

\section{Results and discussion}

\subsection{Microstructures}

The description of the microstructural characteristics of many modern steels must be performed not only at the $\mu \mathrm{m}$ scale but also at the nm-scale. Fig. 2 shows the characteristic grain sizes of different steels. For electrical sheet steels, either grain-oriented or non-oriented, as well as for mild cold-formable low-carbon steels to some extent, large grains are preferred. For most steel applications, however, small grain sizes and homogenous grain-size distributions are of primary interest. Thus far, very small grain sizes can be obtained at the industrial level by thermomechanical processing, which combines heavy hot deformation and the phase transformation of strained super-cooled austenite. The ultrafine-grain steel concept strives for a much finer structure to obtain typical grain sizes on the order of $1 \mu \mathrm{m}$ or even less in nanostructured steels, which are often achieved with extremely

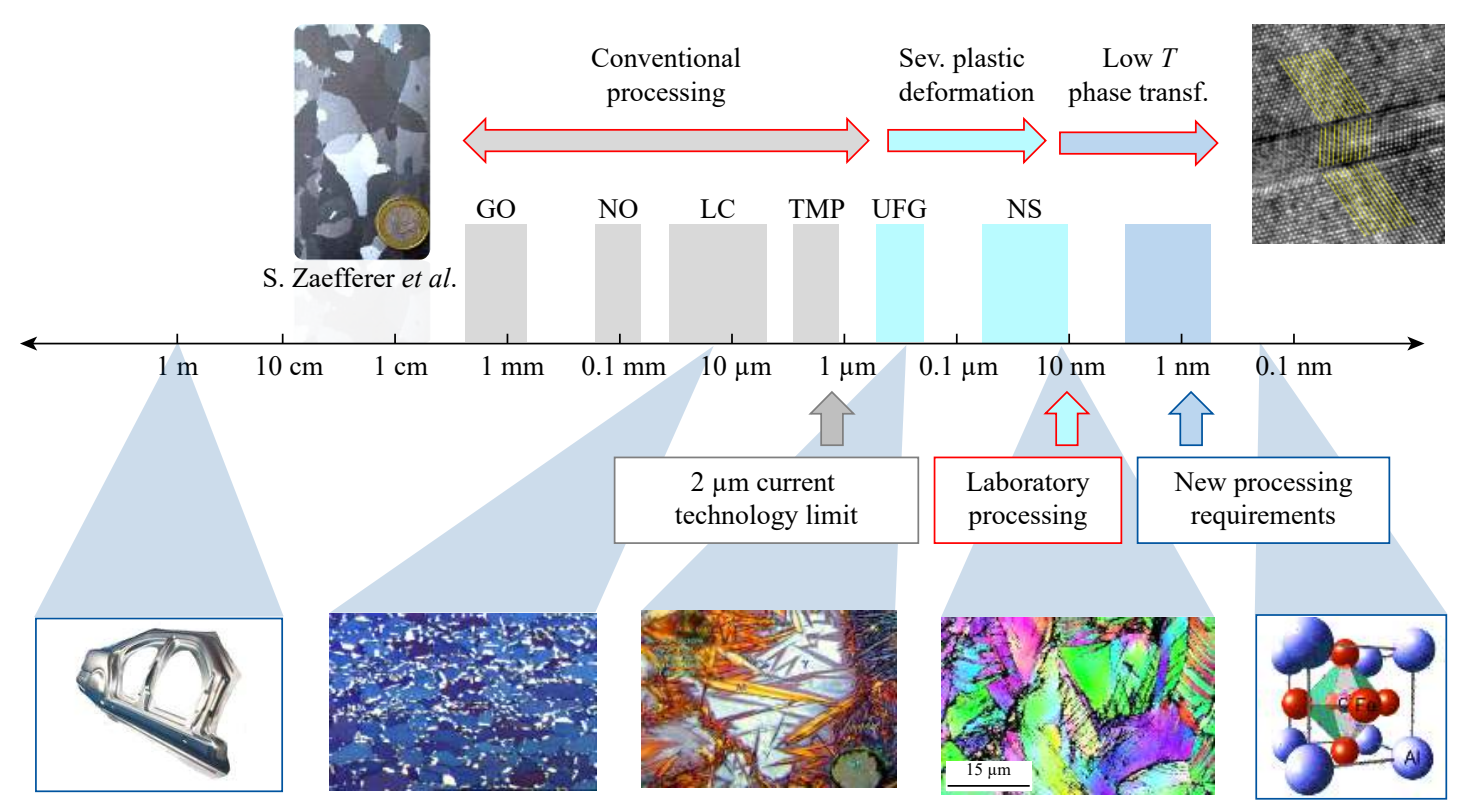

Fig. 2. Grain sizes of different steel products and process routes for the smallest grain sizes. The different scales are illustrated by photos and micrographs. GO-Grain oriented; NO-Non oriented; LC-Low carbon; TMP-Thermomechanical processing; UFG-Ultrafine grain; NS-Nano structured. 
high degrees of deformation. For this purpose, new forming processes have been developed, which are categorized under the term severe plastic deformation (SPD), but these SPD procedures are mostly laboratory processing routes that cannot be transferred to the industrial scale. New options for the development of nanostructured steels rely on the deformation mechanisms of TRIP and TWIP, which continuously refine the microstructure during plastic deformation. At room temperature, strain-induced phase transformations occur and lead to extremely fine microstructures. The TRIP effect depends on the deformation-induced austenite $\rightarrow$ martensite transformation, and the TWIP effect is a consequence of the introduction of nm-scale-deformation-induced twins. In this way, the current minimum grain size of approximately $2 \mu \mathrm{m}$, which is obtained in microalloyed single-phase ferritic steels after thermomechanical rolling on an industrial scale, can be shifted to microstructures on the nm-scale.

At the CRC, different methods for microstructural characterization have been used, which provide structural and analytical information on specific length scales, from structural characterization at the macroscopic scale to analysis with atomic resolution using high-resolution transmission electron microscopy (TEM), scanning transmission electron microscopy (STEM), and atomic probe tomography (APT). Scanning electron microscopy (SEM) and electron backscatter diffraction (EBSD) are used in the analysis of deformed and undeformed steel structures at the nm-scale. Due to the occurrence of transformations at room temperature, an important focus has been the analysis of deformed microstructures and the structural deformation mechanisms when mechanical stresses are applied. Particular attention has been paid to the demarcation between twin formation (TWIP effect) and the martensitic-phase transformation of the material (TRIP effect) during straining, as well as the correlation of these effects with the mechanical properties of the steels. Very finely deformed twins cannot always be resolved with EBSD, which is why electron channeling contrast imaging
(ECCI) is used as a link between the length scales of the EBSD and high-resolution STEM (Fig. 3). ECCI is an imaging technique in SEM based on electron channeling by applying a backscatter electron detector. It is used for the direct observation of lattice defects, for example, dislocations or stacking faults close to the surface of bulk samples and has been proven to be very effective as a tool for the quantitative description of TRIP and TWIP steels [8-9]. High-resolution TEM investigations have provided detailed insights into lattice structures and their defects at the atomic scale. If scanning mode is used in TEM, further structural details can be visualized. In bright field (BF)-STEM, electrons that are scattered are detected when a specimen is being scanned and this imaging mode is dominated by diffraction (Bragg) contrast. Annular dark-field-STEM (ADF-STEM) uses an objective aperture that enables the collection of scattered electrons at intermediate scattering angles in the range of 20-55 mrad on an annular detector. This configuration is well suited for characterizing lattice defects and identifying correlations between dislocation characteristics and segregation. To visualize subtle changes in composition in an APT specimen, high-angle annular dark field-STEM (HAADF-STEM) is the method of choice [10]. These technologies are now used to obtain comprehensive and quantitative information about property-determining structures.

In high-aluminum steels, a special focus is the coherent $\kappa$ phase and its growth and interaction with the lattice defects during plastic deformation. In this way, specific strainhardening phenomena in MBIP steel have been monitored [11]. Fig. 4 shows HAADF-STEM images of the $\kappa$-carbides and their matrix at atomic resolution together with distribution maps of the internal strain, which were determined by evaluating the positions of the atomic columns in the HAADF image. The BF-STEM image at lower magnification shows how the $\kappa$-precipitates hinder the dislocation movement by shearing of the precipitates.

In addition to the structural information, the local chemic-
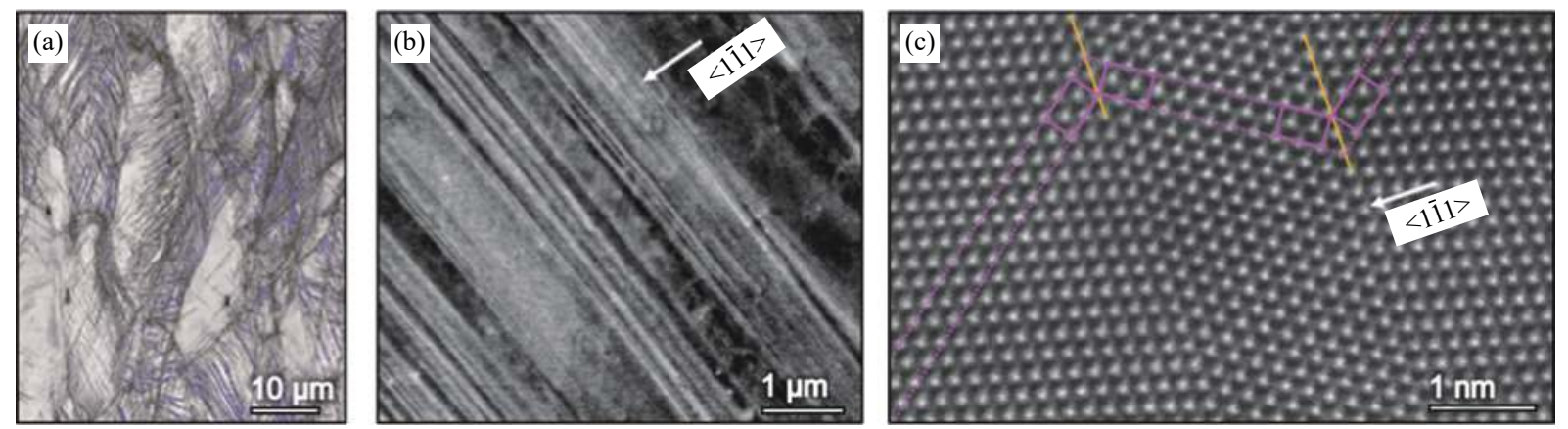

Fig. 3. TWIP steel microstructure after deformation at room temperature, characterized at different length scales. (a) EBSD map (blue-marked lines indicate the $\Sigma 3$ twin boundaries). (b) ECCI representation by orienting the grains in Bragg condition with (1111) $g$ vector (arrow). Individual nano twins can be observed. (c) HAADF-STEM representation in atomic resolution in $<110>$ fce crystallographic orientation, with two twin boundaries, indicated by the orange lines, and the corresponding symmetry of the unit cells (purple). 
al composition in a nanostructured material is of great interest as a means for understanding phenomena such as the segregation of grain boundaries or stacking faults. APT enables 3D measurement of the element distribution with almost atomic spatial resolution and a measurement sensitivity of $\sim 10 \mathrm{ppm}$.

A new experimental setup uses nanobeam diffraction in TEM on atomic probe samples, which enables the measurement of orientation and phase maps with an angular resolution of approximately $1^{\circ}$ and a spatial resolution of approximately $2 \mathrm{~nm}$ (Fig. 5). This information together with precise TEM images of the lattice defects and accurate 3D chemical information obtained using APT provides precise and complete characterizations of all parameters important for understanding nm-scale phenomena, e.g., grain-boundary segregation $[10,12]$.
The investigated HMnS and MMnS feature extremely fine structures, which determine their mechanical behaviors. For example, there are deformation twins with an extension in width of less than $10 \mathrm{~nm}$. This results in very short free dislocation paths on one hand and very short diffusion paths on the other, which enables segregation effects of both interstitial and substitutional alloying elements. Understanding the mechanical behavior of this new material group thus requires use of the sophisticated metallographic inspection methods described above.

\subsection{Deformation mechanisms}

The SFE was identified as the central control variable of the deformation mechanisms of HMnS. The ab-initio and thermodynamic elucidation of the relationship between the chemical composition and the SFE enabled quantitative pre-

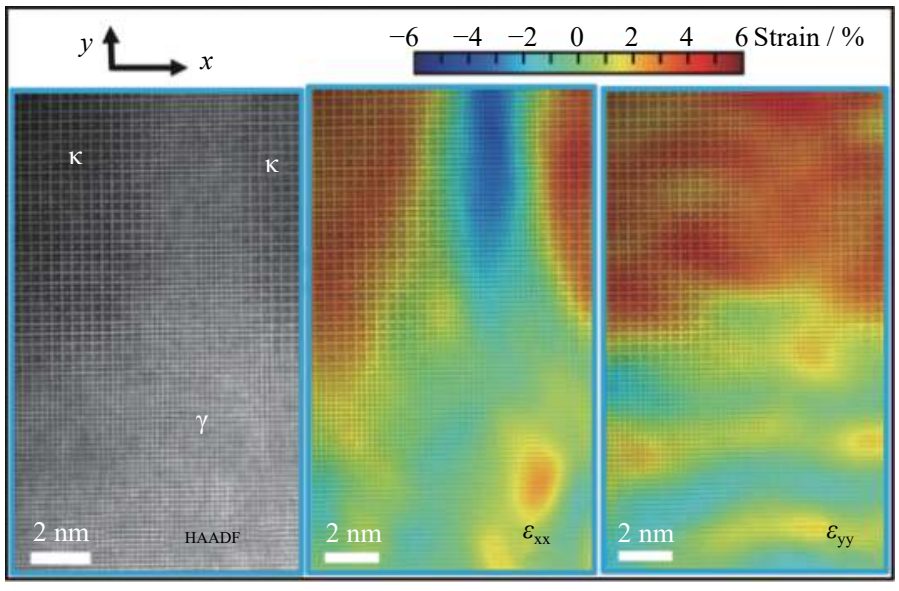

(a)

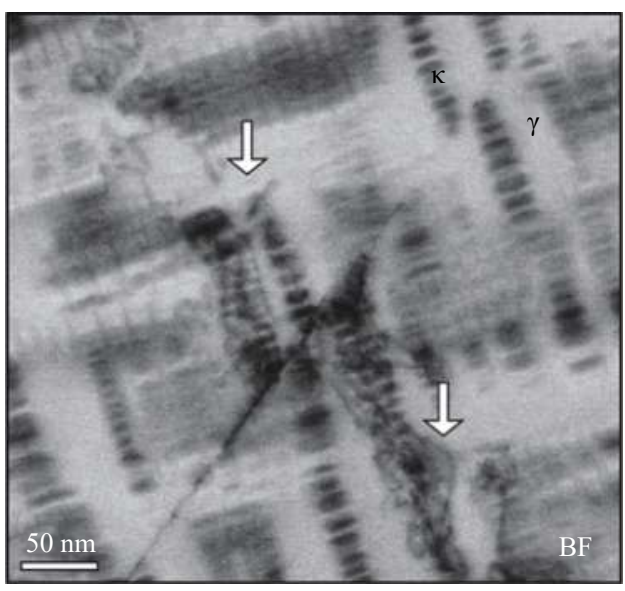

(b)

Fig. 4. STEM images of X120MnAl30-8 steel, which contains $\kappa$-carbides after aging at $600^{\circ} \mathrm{C}$ for $24 \mathrm{~h}$. (a) High-resolution HAADFSTEM image in the crystallographic $<100>$ direction and corresponding maps of the local strain states in two directions $\varepsilon_{\mathrm{xx}}$ and $\varepsilon_{\mathrm{yy}}$, which are defined as the difference between the local interatomic distances and the undistorted reference $\gamma$-matrix. (b) BF-STEM overview display of the deformation structure after 5\% tensile deformation at room temperature. Arrows indicate the dislocation hindrance by k-carbides.

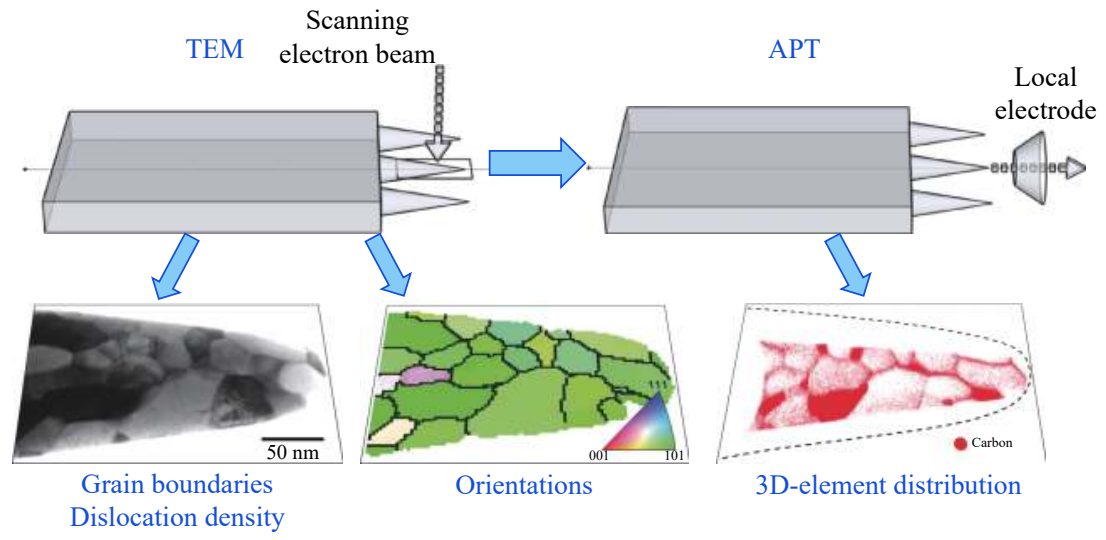

Fig. 5. Schematic illustration of the experimental setup for the correlative TEM/APT measurement: After sample preparation in the two-beam FIB/SEM, it is characterized by TEM and APT. In TEM, the grain-boundary orientations and dislocation densities are measured in imaging mode and the grain orientation by NBD. The APT provides complementary information about the 3D element distribution. FIB—Focused ion beam; NBD—Nanobeam diffraction. 
diction of the active deformation mechanisms as a function of the alloy composition and temperature. Based on our imprecise qualitative understanding at the beginning of the collaborative research, new two-dimensional (2D) and finally 3D mechanism maps were developed for quantitative evaluation (Fig. 6). These enabled the targeted determination of alloy compositions with defined deformation behavior. Two types of maps were developed: composition-composition maps with both axes showing an element content of $\mathrm{C}, \mathrm{Mn}$, or $\mathrm{Al}$, and composition-temperature maps. For the $\mathrm{Fe}-\mathrm{Mn}-\mathrm{A}-\mathrm{C}$ system, the thermodynamically determined area occupied by the $\kappa$-phase was also entered. Finally, the concept of the mechanism map was expanded for MMnS. To do so, the setting of the defined residual austenite levels during intercritical annealing and the formation of carbides had to be taken into account.

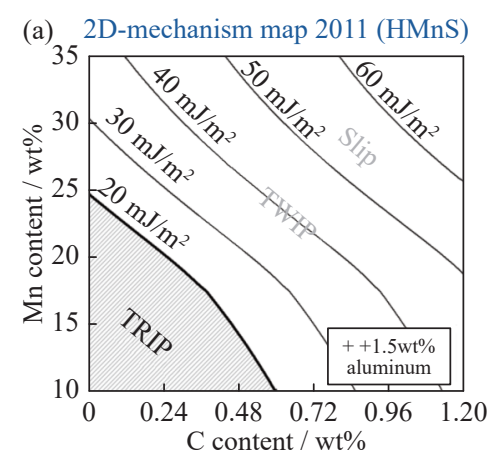

(b) 3D-mechanism map 2014 (HMnS)

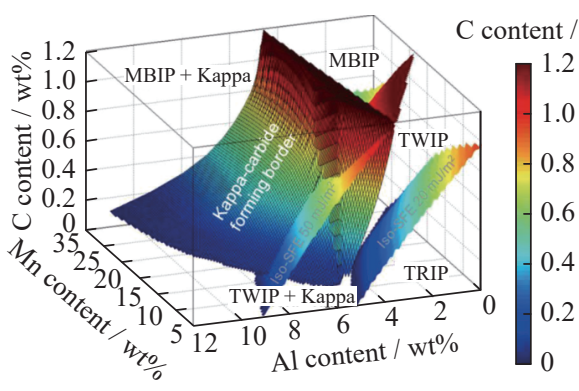

(c) 3D-mechanism map $2019(\mathrm{MMnS})$

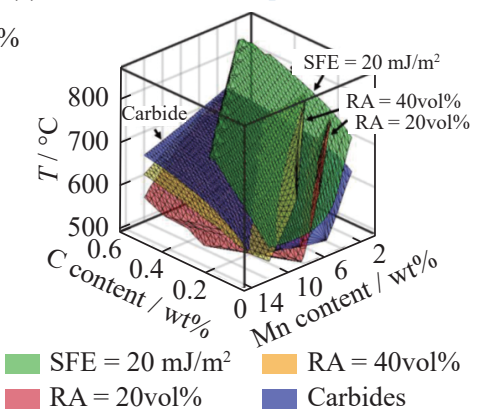

Fig. 6. Descriptions of the dominant deformation mechanisms using mechanism maps. (a) Development during the CRC operational period starting from a 2D-map of the Fe-Mn-C system based on SFE calculation in 2011, to (b) a 3D-map of the Fe-Mn-C-Al system with consideration of SFE and $\kappa-p h a s e$ formation in 2014, to (c) a 3D-map describing MMnS considering SFE, the retained austenite fraction, and carbide formation in 2019.

The basis for calculating the 2D- and 3D-mechanism maps is a "subregular solution" model, with thermodynamic data obtained from the Scientific Group Thermodata Europe (SGTE) database. The basic assumption of this model is that the SFE $(\Gamma)$ corresponds to the free Gibbs energy to form a two-atomic-layer-thick $\varepsilon$-martensite film on the densely packed levels [13-14]. The SFE is calculated according to the equation reported in Ref. [15]:

$$
\Gamma=2 \rho \Delta G_{\text {eff }}^{\gamma \rightarrow \varepsilon}+2 \sigma^{\gamma / \varepsilon}
$$

where $\rho$ is the molar surface density of the $\{111\}$ planes, $\sigma^{\gamma / \varepsilon}$ is the $\gamma / \varepsilon$ interface energy, and $\Delta G_{\text {eff }}^{\gamma \rightarrow \varepsilon}$ is the change in the free Gibbs energy for the $\gamma \rightarrow \varepsilon$ transformation. $\Delta G_{\text {eff }}^{\gamma \rightarrow \varepsilon}$ has two components, the chemical contribution $\Delta G_{\text {chem }}^{\gamma \rightarrow \varepsilon}$ and the magnetic contribution $\Delta G_{\mathrm{mag}}^{\gamma \rightarrow \varepsilon}$ to the change in the free Gibbs energy:

$$
\begin{aligned}
\Delta G_{\mathrm{eff}}^{\gamma \rightarrow \varepsilon} & =\Delta G_{\text {chem }}^{\gamma \rightarrow \varepsilon}+\Delta G_{\mathrm{mag}}^{\gamma \rightarrow \varepsilon} \\
\Delta G_{\mathrm{chem}}^{\gamma \rightarrow \varepsilon} & =\left[\sum X_{i} \Delta G_{i}^{\gamma \rightarrow \varepsilon}+\sum X_{i} X_{j} \Delta \Omega_{i j}^{\gamma \rightarrow \varepsilon}\right]
\end{aligned}
$$

where $X_{i}$ is the molar fraction of element $i$ in the system, $\Delta G_{i}^{\gamma \rightarrow \varepsilon}$ is the change in the free energy of the $\gamma \rightarrow \varepsilon$ transformation, and $\Delta \Omega_{i j}^{\gamma \rightarrow \varepsilon}$ is the interaction energy of components $i$ and $j$.

$$
\Delta G_{\mathrm{mag}}^{\gamma \rightarrow \varepsilon}=R T \ln \left(1+\frac{\beta^{\varphi}}{\mu_{\mathrm{B}}}\right) f\left(\frac{T}{T_{\text {Néel }}^{\varphi}}\right)
$$

Here, $\beta^{\varphi}$ is the magnetic moment, $T_{\text {Néel }}^{\varphi}$ is the Néel temperature of phase $\varphi$, and $\mu_{\mathrm{B}}$ is the Bohr magneton. Further details on the development of the mechanism maps are provided by Saeed-Akbari et al. [16]. All information about the SFE in this work is based on these calculations.

Fig. 6 presents a 3D-mechanism map for the Fe-Mn$\mathrm{C}-\mathrm{Al}$ system for the content range of $0-12 \mathrm{wt} \% \mathrm{Al}, 5 \mathrm{wt} \%-$ $35 \mathrm{wt} \% \mathrm{Mn}$, and $0-1.2 \mathrm{wt} \% \mathrm{C}$ at room temperature. Based on the thermodynamically determined content-dependent SFEs, the mechanism map shows the dominant deformation mechanisms in the $3 \mathrm{D}$ concentration space. The addition of $\mathrm{Al}$ pushes the SFE to higher values on one hand and promotes the elimination of the $\kappa$-phase on the other. With SFEs of more than $50 \mathrm{~mJ} / \mathrm{m}^{2}$, dislocation slip is the preferred activated deformation mechanism. A special feature here is the occurrence of planar dislocation activity in the form of microbands, although the high SFE tends to favor the cross slip of dislocations. Obviously, these microbands are related to the occurrence of the $\kappa$-phase. The mechanism maps for MMnS must mainly consider the probability of carbide precipitates and the phase fractions of austenite with respect to the local chemical composition. The latter is controlled by non-equilibrium partitioning of the alloy elements during complex annealing treatments.

\subsection{Strain-hardening behavior}

On the basis of these mechanism maps, alloy systems with different strain-hardening behaviors were developed and manufactured in response to the activated deformation mechanisms using the abovementioned laboratory casting and rolling processes. $\mathrm{HMnS}$ are either metastable materials in 
which the microstructure is stable upon cooling but not under loading, e.g., TRIP steels, or materials that remain stable even after loading but show an extensive twin formation during plastic deformation, e.g., TWIP steels. TRIP steels undergo multiple martensitic transformations from fcc austenite to hcp martensite and finally to bcc martensite upon loading.

Fig. 7 shows that the strain-hardening behavior can be controlled by specifically setting the SFE. Steels with different predominant deformation mechanisms show significant differences in their strain-hardening behaviors. X60MnAl171 steel is dominated by either TRIP or TWIP, depending on the test temperature. MBIP X120MnAl30-8 steel is documented with and without $\kappa$-phase precipitation. This is accomplished by combining the deformation mechanisms (e.g., multiphase and TRIP in MMnS, precipitation hardening using $\kappa$-carbides and planar glide in MBIP steels) to enable the development of expanded property profiles. With these new steels, the strain hardening can be set largely independent of the strength level; i.e., the slope of the flow curve can be controlled independent of the yield point by choosing the deformation mechanisms. This feature is of great interest for further processing of the sheets by cold-forming processes. The steels in Fig. 7 were selected as examples to show that the TRIP effect is often associated with a very steep gradient in the flow curve, and sometimes exhibits a turning point as a result of the deformation-induced martensite formation. TWIP steels often have a slightly lower but very uniform slope, which leads to a very high uniform elongation in tensile tests. However, the observed post-uniform elongation is minimal, and the uniform and total elongation often coincide. The MBIP effect is associated with a wide range of mechanical properties, depending on whether the alloying elements are dissolved or precipitate as $\kappa$-phase. The formation of the $\kappa$-phase leads to a significant increase in the yield strength with a lesser but still good overall elongation, with respect to the strength level. However, the strain hardening that occurs is more normal to that of high-strength steels. After all, MMnS are of particular interest as a new group of

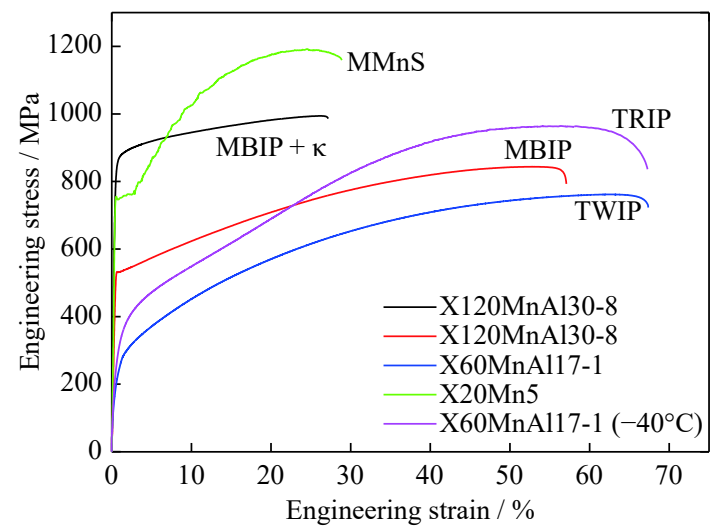

Fig. 7. Engineering stress-strain curves of various investigated steels. high-strength grades whose mechanical behaviors are characterized by a mixture of soft and hard phases. One characteristic of these steels is a certain amount of residual austenite, which indicates the TRIP effect and thus leads to pronounced strain hardening, occasionally also combined with serrated yielding. In addition, there are clear connections between the process control in press forming and the activation or suppression of certain deformation mechanisms. As such, the deformation behavior of $\mathrm{HMnS}$ or $\mathrm{MMnS}$ can be varied over a wide range.

\subsection{Temperature dependency of mechanical properties}

The mechanical properties of HMnS depend primarily on their active deformation mechanisms, which vary with temperature due to the change in the SFE. Fig. 8 shows plots of the variations of mechanical properties, including yield strength (YS), ultimate tensile strength (UTS), uniform elongation (UE), and total elongation (TE) as a function of temperature and SFE for X60Mn22 steel. The figure shows both the temperature ranges where the deformation mechanisms SLIP, TWIP, and TRIP are active and that at which serrated flow occurs due to dynamic strain aging (DSA) [17-18].

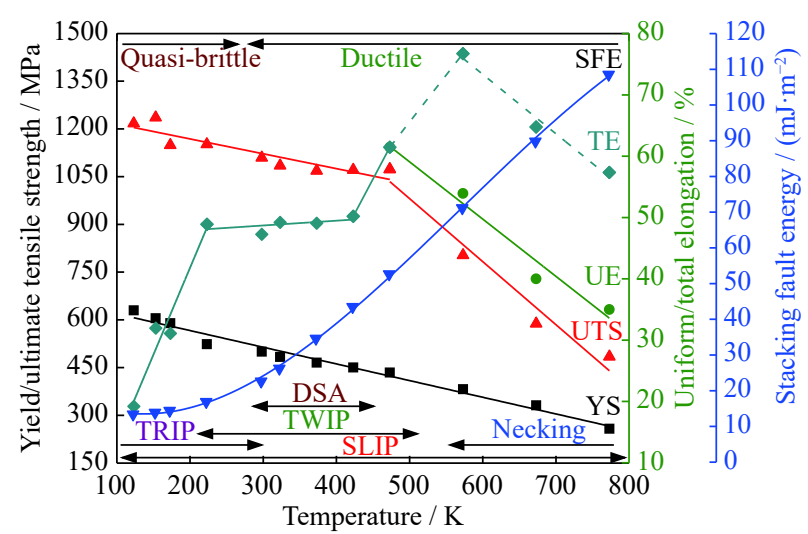

Fig. 8. Mechanical properties as a function of test temperature for X60Mn22 steel. The ranges for the deformation mechanisms TRIP, TWIP, SLIP, and DSA are indicated, as is the occurrence of necking prior to failure. Reprinted from Int. $J$. Plast., 104, M. Madivala, A. Schwedt, S.L. Wong, F. Roters, U. Prahl, and W. Bleck, Temperature dependent strain hardening and fracture behavior of TWIP steel, 80-103, Copyright 2018, with permission from Elsevier.

The deformation mechanisms of the X60Mn22 steel strongly depend on temperature. Below room temperature, the TRIP effect dominates with the formation of $\alpha^{\prime}$-martensite. The lower is the test temperature, the greater is the volume fraction of martensite, such that the elongation values decrease with decreasing temperature. In addition to the formation of martensite, isolated deformation-induced twins can also be detected, but these are not decisive at temperatures $<298 \mathrm{~K}$. Twinning and dislocation glide are the domin- 
ant deformation mechanisms at temperatures above $298 \mathrm{~K}$, with the twin fraction increasing with temperature up to the transition temperature of $473 \mathrm{~K}$. No twins are evident at above $473 \mathrm{~K}$, with the deformation then dominated by dislocation slip.

At the lowest investigated temperature of $123 \mathrm{~K}$, the material has a YS of approximately $600 \mathrm{MPa}$ and a UTS of approximately $1200 \mathrm{MPa}$. The YS and the UTS values decrease almost linearly with increasing temperature. Above $473 \mathrm{~K}$, the UTS drops sharply when the temperature is increased. At temperatures between 673 and $773 \mathrm{~K}$, the UTS ranges between 580 and $480 \mathrm{MPa}$, which is approximately half that at $298 \mathrm{~K}$. The TE of the material is approximately $20 \%$ at $123 \mathrm{~K}$, then increases with temperature to a plateau of about $50 \%$ and remains almost constant at temperatures between 233 and $373 \mathrm{~K}$, at which twinning occurs simultaneously in combination with DSA and dislocation glide. The TE increases to $>60 \%$ at temperatures above $423 \mathrm{~K}$. However, the UE, which is very similar to the TE at lower temperatures, decreases to almost $40 \%$ at temperatures above $673 \mathrm{~K}$. As a consequence, it can be concluded that particularly attractive strength-formability combinations are associated primarily with the TWIP phenomenon. DSA in low-carbon steels is usually considered to be detrimental to formability. However, the DSA effect observed in HMnS at certain temperature ranges is not reflected by a drop in elongation.

\subsection{Failure modes}

High-manganese TWIP steels generally exhibit ductile failure over a wide range of temperatures and strain rates, The fracture surface is controlled by different active deformation mechanisms: quasi-brittle islands along with fine dimples at low temperatures due to $\varepsilon$-martensite transformation, shallow dimples at moderate temperatures that occur with DSA and twinning, and larger dimples with elliptical voids at $\mathrm{MnS}$ inclusions at higher temperatures. Since twinning is the most dominant deformation mechanism for a wide range of temperatures, ductile fracture with fine dimples are commonly observed.

A specific feature of TWIP steel specimens is that no necking is observed prior to failure, which means that both elongation values of the tensile test, i.e., UE and TE, coincide at temperatures below $500 \mathrm{~K}$. The observed serrated flow between approximately 300 and $450 \mathrm{~K}$, caused by DSA, leads to strain localization in shear bands and finally to local plastic instability. During tensile tests, shear bands nucleate and propagate from one specimen shoulder to the other throughout the duration of the test, leading to an inhomogeneous strain distribution with localization of plastic strain in the shear bands. In the course of tensile tests, several shear bands emerge one after the other, the running speeds of which gradually decrease as a result of the strengthening of the material. Failure of the material occurs when the motion of the shear bands is strongly retarded, e.g., by interaction with a second shear band, such that the band velocity approaches zero. Microcracks are mainly initiated at the intersection of twin bundles with grain boundaries or triple junctions. These microcracks propagate rapidly along the grain boundary, resulting in trans-granular but still ductile fracture. The observed mechanisms of microcrack formation in TWIP steels are categorized into three failure modes, which are schematically illustrated in Fig. 9. Failure mode I is an inclusion-controlled mechanism that occurs in the presence of large inclusions. Failure mode II occurs when cracks are initiated at surface defects or edges when the intersecting shear bands stop their movement. At the microscopic level, failure mode III is mostly observed when twin bundles are intersected by grain boundaries, which leads to stress concentrations and inter-granular microcracks that are aligned along the grain boundary [19].

Of course, the degree of internal cleanliness also plays a role in failure behavior. For example, some isolated microcracks can be observed on $\mathrm{MnS}$ inclusions, which have appeared during an early stage of deformation. Surprisingly, these microcracks do not evolve into cracks and are not relevant to the global behavior of the test specimen.

Only the presence of very coarse inclusions can lead to very local stress concentrations that can cause localized rupture. The few and small inclusions in materials with a high degree of internal cleanliness have no impact on failure development as the microcracks that may occur are healed by the high local strain-hardening potential of the matrix.

There are also many instances where a boundary is shared by grains, which distinguish their deformation mechanisms, e.g., grains that deformed by twinning and their neighboring grains by slip. The interception of slip band extrusions by twins at the grain boundary results in incompatible deformation, which leads to microcrack formation. The predominant inter-granular fracture in tension suggests that segregation of the alloying or impurity atoms, which might occur during high-temperature annealing, may also affect the final damage. In any case, the observed very small and very shallow dimples on the fracture surface indicate that numerous cracks initiate, obviously at intersections with austenite grain boundaries, and immediately lead to mesoscopic crack growth when the strain-hardening potential of the matrix is exhausted. The typical dimple size in $\mathrm{HMnS}$ is about $2 \mu \mathrm{m}$ compared to $>5 \mu \mathrm{m}$ in conventional low-carbon steels. Thus, no necking of the tensile test specimens is observed and the macroscopic crack propagates perpendicular to the tensile direction.

To analyze the influence of multiaxiality on the formability of different AHSS, tensile, notch-tensile, and shear-tensile tests were performed on TWIP1000 steel, along with the dual-phase DP780 steel and the low-alloy TRIP800 steel as reference materials. Multiaxiality was found to have a pro- 
(a) Failure mode 1: inclusions controlled
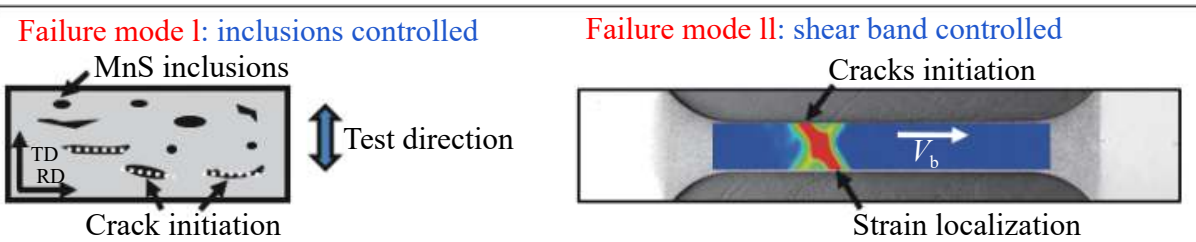

Failure mode 11l: interacting deformation mechanisms controlled
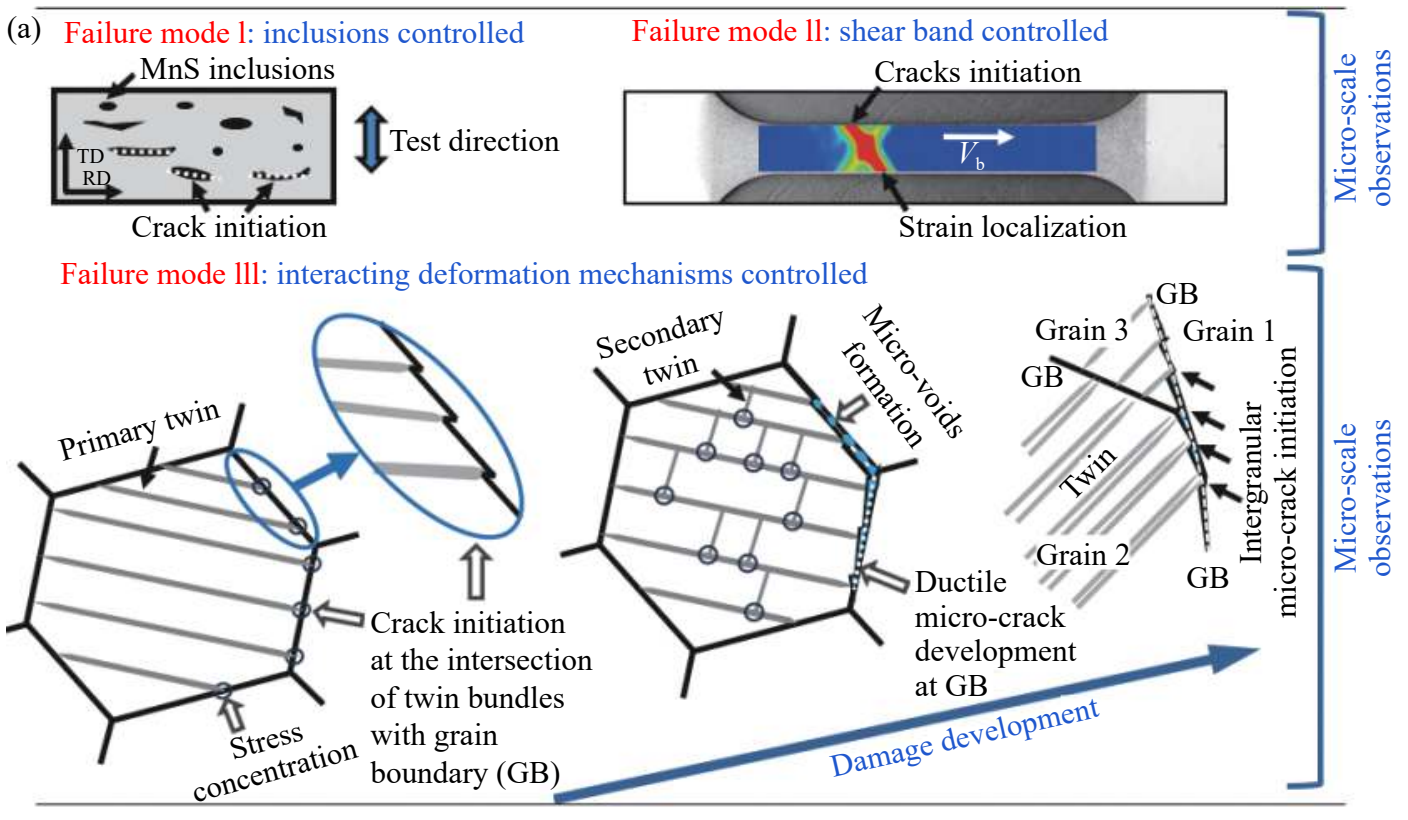

(b)

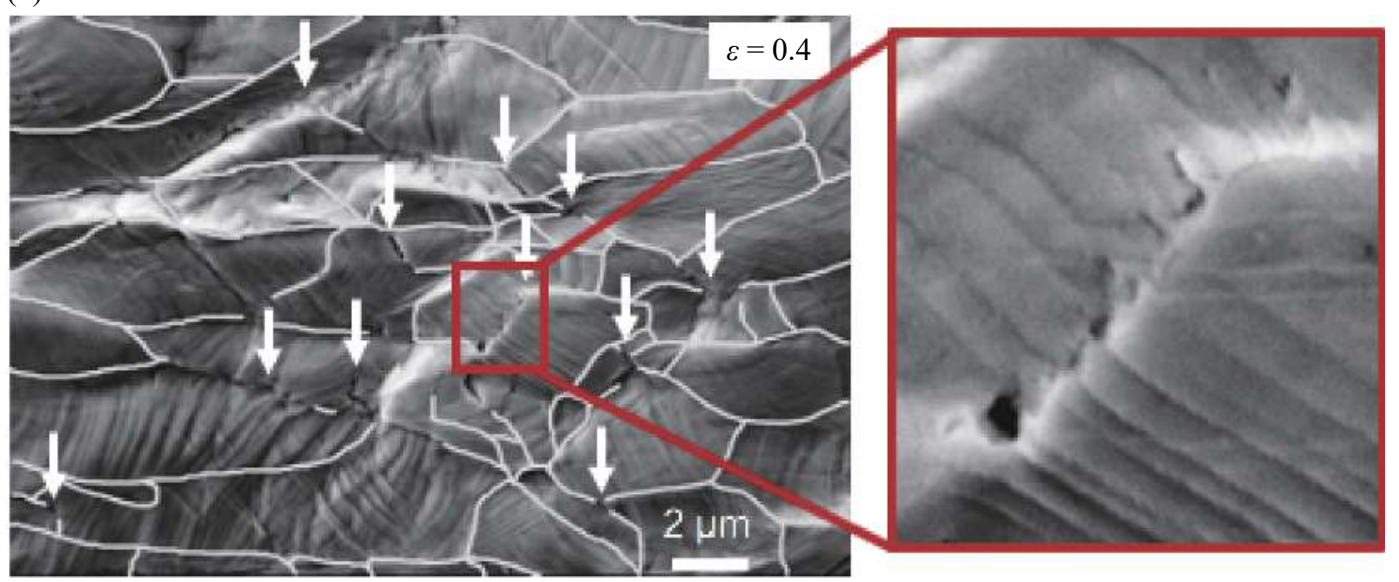

Fig. 9. Failure modes in TWIP steels: (a) schematic illustration of the different failure modes; (b) SEM images of the intersection of twin bundles and a grain boundary, showing void formation along the grain boundary. The white arrows indicate the multitude of crack initiation points that occur simultaneously. Reprinted from Int. J. Plast., 115, M. Madivala, A. Schwedt, U. Prahl, and W. Bleck, Anisotropy and strain rate effects on the failure behavior of TWIP steel: A multiscale experimental study, 178-199, Copyright 2019, with permission from Elsevier.

nounced influence on the failure behavior (Fig. 10), with the highest failure strain values obtained in uniaxial tensile tests and significantly lower values in plane strain conditions or especially in the case of shear deformation. By correlating the experimental results with fracture surface analysis, the influence of void formation could be determined. The fracture mode was found to be ductile under all loads, with significant differences in the fracture surface appearance. Faster void growth and failure was observed in samples with higher multiaxiality, i.e., the notched samples. It is striking that the HMnS exhibit almost no post-necking behavior under all loads, which can be explained by their late formation of voids in the shear bands, which in turn is controlled by the twin density. This behavior can also be described in simulation calculations with a phenomenological twin density-based damage model.

In principle, Fig. 10 shows the fundamental superiority of the TWIP1000 steel compared to the reference steels under positive triaxialities, which is the maximum in tensile tests but not with pure shear. A significant difference is the failure without necking of the TWIP1000 steel, whereas the DP780 and TRIP800 grades show a classic behavior with uniform stretching and subsequent post-necking behavior. Thus, in the event of failure, the local elongation at break is greater than the global one. With low triaxiality, shear failure occurs, which is evident on the fracture surface in the form of shear lips. Under this failure mode, there is no difference between the steels. 


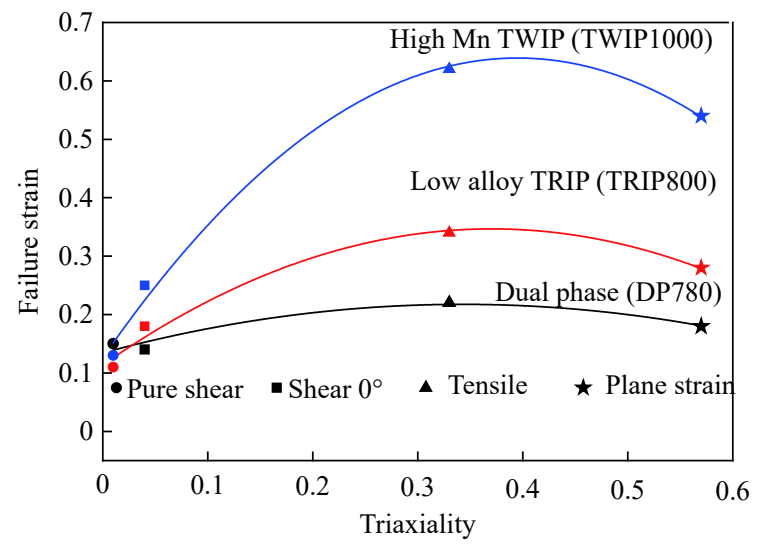

Fig. 10. Influence of stress triaxiality on the failure behavior of X60Mn22 steel (TWIP1000) compared to the dual-phase DP780 steel and the low-alloy TRIP800 steel.

\subsection{Impact of short-range ordering on mechanical prop- erties}

Inhomogeneous serrated flow at elevated temperatures is often associated with DSA. The dislocation movement is continuously blocked by fast-moving interstitial atoms that form a Cottrell cloud around the dislocation. Inhomogeneous plastic flow in $\mathrm{HMnS}$ cannot be adequately described by this phenomenon, since it is observed at room temperature, where the diffusion of carbon atoms and thus the formation of Cottrell clouds are unlikely. The high content of alloying elements in $\mathrm{HMnS}$ results in the frequent interaction of the various alloying elements. Due to a strong interaction between the interstitially dissolved carbon and the surrounding substitutionally dissolved atoms, there are atomic arrangements that are energetically preferential. Ab-initio calculations using density-functional theory show that this is the case for manganese atoms as nearest neighbors and aluminum atoms as next-nearest neighbors. Accordingly, the distribution of carbon in the austenitic matrix is guided by the presence of alloying elements rather than being completely random. This leads to the formation of short-range ordered zones with just one or a few elementary cells.

As an example, short-range ordering (SRO) phenomena have been observed in $\mathrm{HMnS}$, which are interpreted based on the preferential location of carbon in manganese-rich octahedral gaps. Ab-initio calculations have shown that carbon atoms seek a manganese environment to minimize the local lattice distortion.

To establish a quantitative approach to determining the preferred atomic arrangement of $\mathrm{C}$ in $\mathrm{Fe}-\mathrm{Mn}$ alloys, an fcc super - cell containing $16 \mathrm{Mn}$ and $16 \mathrm{Fe}$ atoms was used for simulation. The calculation considered the entire $\mathrm{Fe}_{16} \mathrm{Mn}_{16}$ structure containing a single carbon atom in one of the ten possible octahedral environments without changing the metal sublattice. This procedure ensures that energy differences are only because of different carbon environments and not because of various metal-metal interactions. This supercell and its ten octahedral gaps correspond to ten different conformations within $\mathrm{Fe}_{16} \mathrm{Mn}_{16} \mathrm{C}$ for comparison with the carbon - free binary system. Fig. 11 shows the reaction energy, which is calculated using

$\Delta E_{\mathrm{R}}=E\left(\mathrm{Fe}_{16} \mathrm{Mn}_{16} \mathrm{C}\right)-E\left(\mathrm{Fe}_{16} \mathrm{Mn}_{16}\right)-E(\mathrm{C})$

as a function of the coordination number $(\mathrm{CN})$ of the $\mathrm{Mn}$ atoms. $E(\mathrm{C})$ is the total energy of carbon in its reference state, the graphite structure. Additionally, the energy differences relative to the most stable structure are shown (right axis in Fig. 11). Obviously, the larger is the number of $\mathrm{Mn}$ atoms coordinating the $\mathrm{C}$ atom, the more stable is the compound. Moreover, this trend is almost linear if only the most stable isomer belonging to each $\mathrm{CN}$ is considered [20-21].

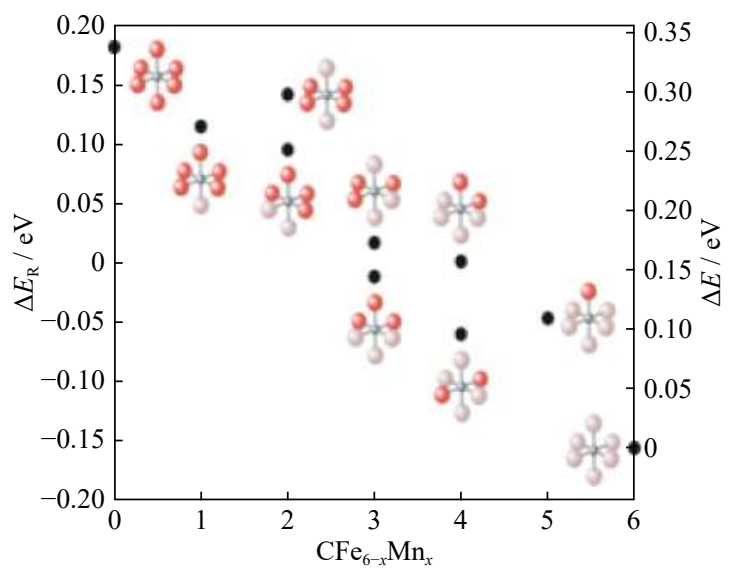

Fig. 11. Reaction energies $\left(\Delta E_{\mathrm{R}}\right)$ and energy differences relative to the most stable conformation $(\Delta E)$ as a function of octahedra with different coordination $\mathrm{Fe}_{6-x} \mathrm{Mn}_{x}$ for a 32-atom-containing face-centered-cubic supercell $\left(\mathrm{CFe}_{6-x} \mathrm{Mn}_{x}\right.$ represents coordination $\mathrm{Fe}_{6-x} \mathrm{Mn}_{x}$ ). J. von Appen and R. Dronskowski, Steel Res. Int., 82, 101-107(2011) [20]. Copyright John Wiley and Sons. Reproduced with permission.

An SRO is an effective barrier for preventing dislocation movements in high-alloy materials. When examining the influence of SRO on the YP, it becomes clear that SRO also hinders the dislocation movement in HMnS. If a system is stabilized by SRO, the degree of stabilization will be proportional to the energy difference between the SRO system $\left(E_{\mathrm{SRO}}\right)$ and the random system $\left(E_{\mathrm{random}}\right)$. By ab-initio calculations and using the McLellan cell model [22], the energy difference for different chemical compositions and temperatures has been determined [23]. The stress required to overcome SRO $\left(\sigma_{\mathrm{SRO}}\right)$ is described by the energy difference as follows:

$\sigma_{\mathrm{SRO}}=M\left(E_{\text {random }}-E_{\mathrm{SRO}}\right) / b^{3}$

where $b$ is the size of the Burgers vector and $M$ is the Taylor factor. The YS $\left(\sigma_{\mathrm{y}}\right)$ can then be specified as 
$\sigma_{\mathrm{y}}=\sigma_{0}+11.3 d^{-1 / 2}+\sigma_{\mathrm{ss}}+\sigma_{\mathrm{SRO}}$

The first constant term $\sigma_{0}$ describes the Peierls friction of a pure face-centered cubic Fe crystal and the second term describes the Hall-Petch hardening, taking into account the grain size $(d)$ [24]. If solid solution strengthening $\left(\sigma_{\mathrm{ss}}\right)$ is also considered, the impact of the contribution of SRO to the YS $\left(\sigma_{\mathrm{SRO}}\right)$ can be estimated. For different HMnS, $\sigma_{\mathrm{SRO}}$ has been shown to be as high as about $65 \mathrm{MPa}$, providing a non-negligible $20 \%$ contribution to the YS. Thus, the impact of alloying elements in HMnS must consider the contribution of the specific element to the change in the SFE, and thus the selection of the dominant deformation mechanisms, along with the solid solution strengthening effect and the impact on the occurrence of SRO.

Various effects in HMnS have been linked to the impact of SRO. The pronounced planar dislocation slip has been theorized to be a result of a glide-plane softening that occurs when SRO zones are cut [25-26]. Additionally, bakehardening heat treatments have yielded significant strength increases when used in HMnS. In addition, it is likely that

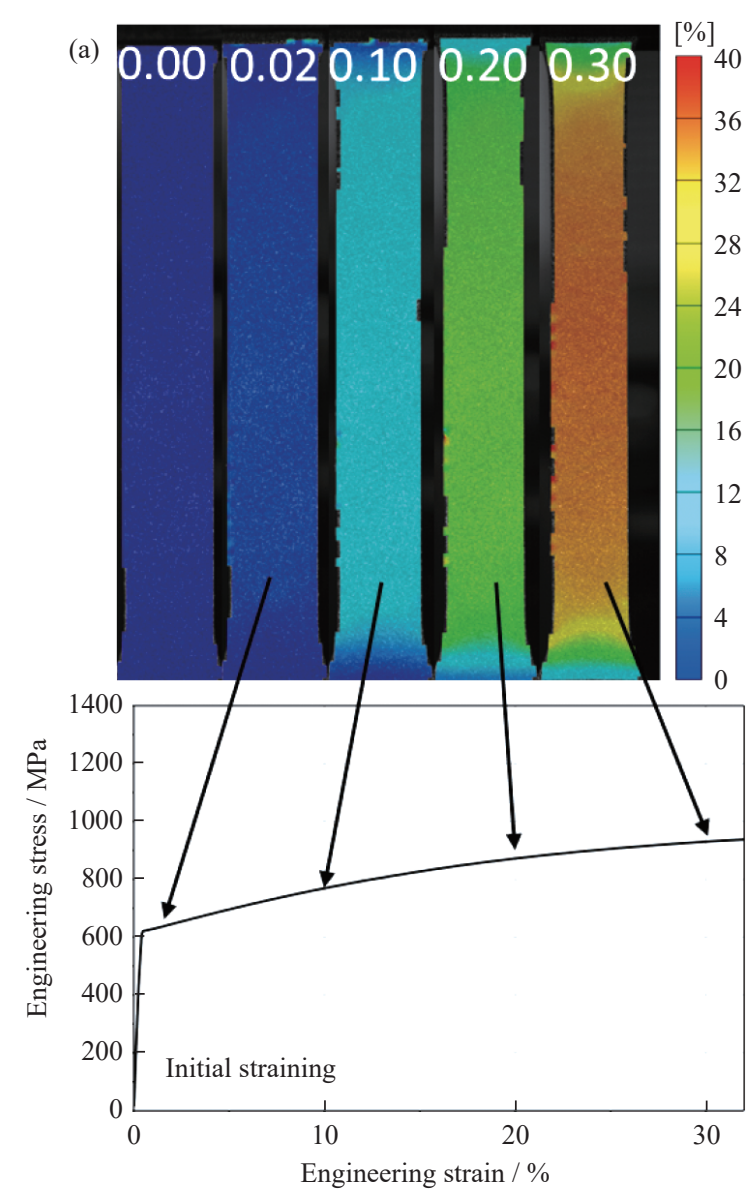

SRO can be restored at relatively low temperatures like room temperature via changes in the short-range C-position, which are favored by the interaction of carbon with either stacking faults or dislocation cores. Accordingly, it is possible for dislocations to be blocked again by a restored SRO during deformation, such that DSA can occur.

Fig. 12 shows a comparison of the local deformation behaviors of X40MnCrAl19-2-1 steel during straining in a tensile test at the initial state and after a $0.2 \%$ prestrain and bake-hardening treatment $\left(170^{\circ} \mathrm{C} / 20 \mathrm{~min}\right)$. During the tensile test, the local strain distribution was determined by digital image correlation. The local strain is presented by color, and the global strain applied in the test is indicated by the value at the top of each picture. In the beginning of the test, the steel was found to deform homogeneously even at the very high strain of 0.30 , and neither strain localization nor pronounced yielding or serrated flow is observed. In contrast, a pronounced yielding is observed after prestraining and bakehardening treatment. This coincides with two shear bands that nucleate at the specimen shoulder and then propagates

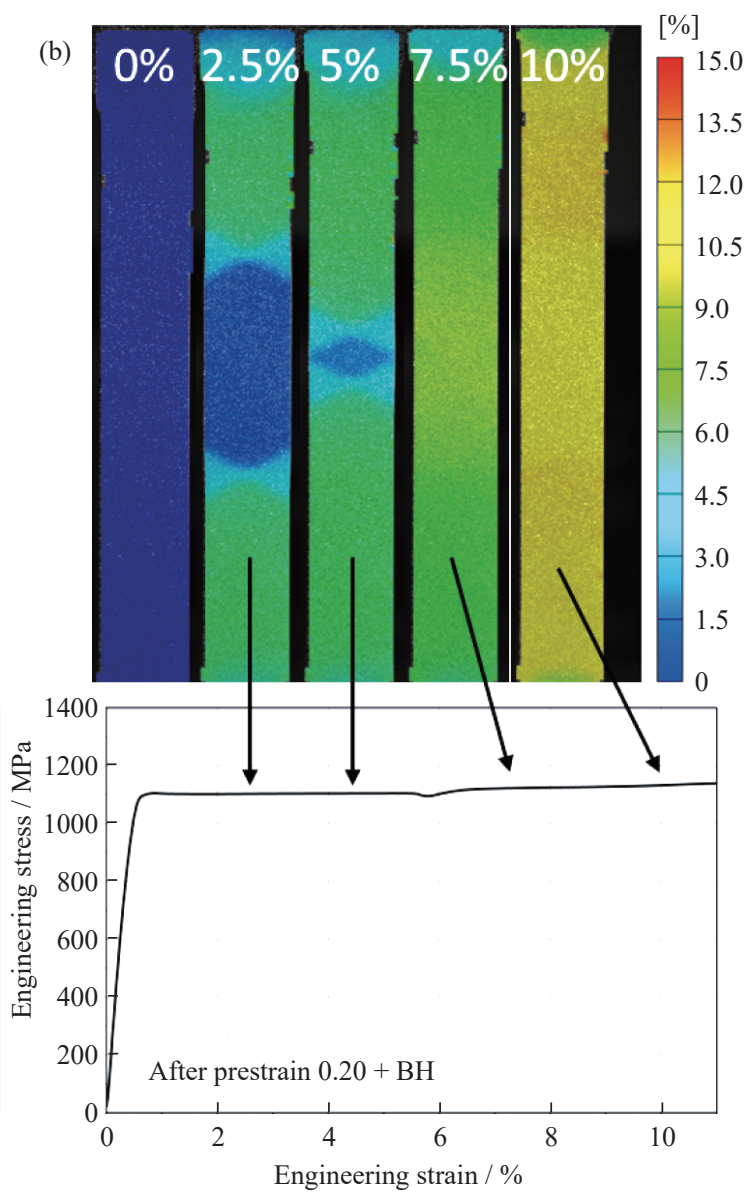

Fig. 12. Local strain distributions of tensile test specimens and engineering stress-strain curves of X40MnCrAl19-2-1 steel: (a) the annealed state; (b) the prestrained state. The local strain is indicated by color. S. Wesselmecking, W.W. Song, Y. Ma, T. Roesler, H. Hofmann, and W. Bleck, Steel Res. Int., 89, art. No. 1700515 (2018) [27]. Copyright John Wiley and Sons. Reproduced with permission. 
toward the center length of the gauge. After 5\% global strain, the shear bands converge in the center of the specimen. As the tensile test progresses, the deformation bands disappear and the strain distribution becomes homogeneous. No further bands are detected until specimen fracture [27-28].

Due to the nanoscale nature and coherency of the SRO zones within the matrix, the existence of SRO is very difficult to prove experimentally. However, small-angle neutron scattering experiments [29] and Mössbauer spectroscopy [25] have been used to indicate the presence of SRO zones. Additionally, TEM experiments have revealed intermediate stages between $\kappa$-carbides and SRO zones, so called longrange ordered zones between 2 and $5 \mathrm{~nm}$. These nano-sized Mn-C SRO clusters form during bake-hardening treatment and contribute to the increase in the YS and to inhomogeneous deformation. It is noted that these $\mathrm{Mn}-\mathrm{C}$-clusters can form even at very low temperatures. Aging was observed even at room temperature on several heavily prestrained samples. This bake-hardening effect can therefore only be used if room-temperature aging can be avoided.

\subsection{Possible areas of application}

New steel design concepts utilize the alloying element manganese for stabilizing the fcc phase and adjusting the SFE. This makes possible the selection of different deformation mechanisms, which can change the strain-hardening behavior in a range of variations not previously known.

This is mainly because of the occurrence of stress-controlled or strain-induced low-temperature transformations of the austenite that provide the basis for new steel groups. These steels are interesting for use in a number of applications. Their potential for use in sheet-metal forming, in the manufacture of forged products, and for wear resistance have been examined intensively. In this paper, the focus has been on advanced high-strength sheet steels chosen for their high cold formability.

Fig. 13 shows an overview of high-strength cold-formable steels alloyed with manganese. The term AHSS refers to multiphase steels with a predominantly ferritic matrix (AHSS 1.G), to the HMnS discussed in this paper with a single-phase austenitic matrix (AHSS 2.G), and to the area of multiphase $\mathrm{MMnS}$ with a martensitic matrix and high metastable austenite contents (AHSS 3.G), which are of particular industrial interest for the future. The economy index ECO (UTS $\times$ TE) is an overview parameter for the performance of steel concepts. Compared to AHSS 1.G, which include the most prominent dual-phase steels and low-alloy TRIP steels, AHSS 2.G provides an extraordinary balance of strength and formability. Depending on the SFE, different deformation modes, either TRIP or TWIP, result in different microstructures after forming. The disadvantage of AHSS is the high-alloy content and the corresponding high costs for its production. As a compromise, AHSS 3.G was developed with a significantly lower alloy content, yielding attractive mechanical properties but high demands on process control, especially during annealing [30].

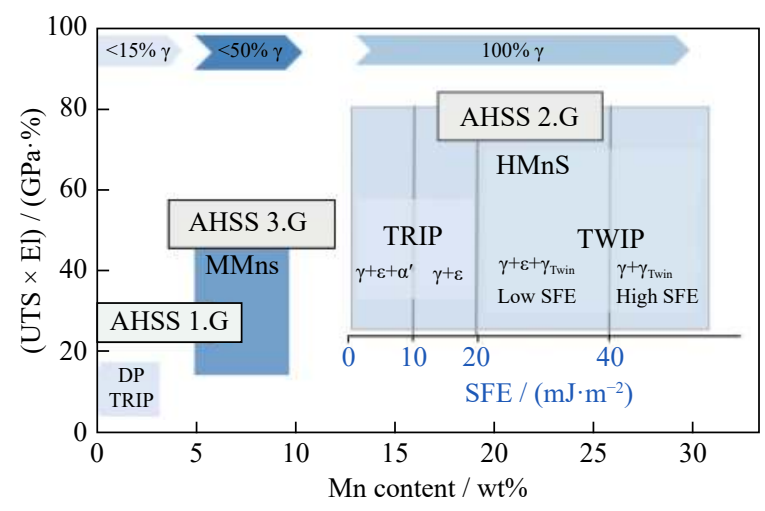

Fig. 13. Mechanical properties of new steel concepts with high-Mn contents. Schematic illustrations of the typical Mn ranges and property balances for advanced high-strength steels of generations 1, 2, and 3 . The austenite content $\gamma$ is indicated.

HMnS are expected to be attractive for automotive applications. The specific requests and targets for this application field include: (1) high strength, lightweight design by reduced sheet thickness; (2) high formability, complex geometries with fewer forming steps; (3) high energy absorption, increased safety when used in crash-relevant components; (4) cost reduction, replacement of cost-intensive materials (Al, $\mathrm{Mg}$, stainless steel) or cost-intensive processes (hot pressing).

In addition to these automotive-related applications for sheet-metal materials, the literature also mentions the use of these steels in the forging industry [31], in new concepts of stainless high-strength steels [32], in cryogenic applications [33], for wear and tear [34], and as alternative damage-tolerant materials for use in additive manufacturing processes [35].

\section{Conclusion}

The results presented here show that modern structural materials with very attractive properties can be produced from steels alloyed with manganese. These steels require characterization at the nm-scale to provide a quantitative understanding of their relevant microstructural features. They can be developed with a wide range of mechanical properties by the activation of different deformation mechanisms, and are affected by the strong interaction among the alloying elements as well as with various crystal defects, e.g., short-range ordering phenomena are important parameters for the control of the mechanical properties.

\section{Acknowledgement}

The financial support of the Deutsche Forschungsge- 
meinschaft (DFG) within the Collaborative Research Centre 761 "Steel - $a b$ initio; quantum mechanics guided design of new Fe based materials" is gratefully acknowledged.

Open Access funding enabled and organized by Projekt DEAL.

Open Access This article is licensed under a Creative Commons Attribution 4.0 International License, which permits use, sharing, adaptation, distribution and reproduction in any medium or format, as long as you give appropriate credit to the original author(s) and the source, provide a link to the Creative Commons licence, and indicate if changes were made. The images or other third party material in this article are included in the article's Creative Commons licence, unless indicated otherwise in a credit line to the material. If material is not included in the article's Creative Commons licence and your intended use is not permitted by statutory regulation or exceeds the permitted use, you will need to obtain permission directly from the copyright holder. To view a copy of this licence, visit http://creativecommons.org/licenses/by/4.0/.

\section{References}

[1] O. Bouaziz, S. Allain, C.P. Scott, P. Cugy, and D. Barbier, High Manganese austenitic twinning induced plasticity steels: A review of the microstructure properties relationships, Curr. Opin. Solid State Mater. Sci., 15(2011), No. 4, p. 141.

[2] H. Kim, D.W. Suh, and N.J. Kim, Fe-Al-Mn-C lightweight structural alloys: A review of the microstructures and mechanical properties, Sci. Technol. Adv. Mater., 14(2013), No. 1, art. No. 014205.

[3] B.C. De Cooman, K.-G. Chin, and J.K. Kim, High Mn TWIP Steels for Automotive Applications, [in] New Trends and Development in Automotive System, IntechOpen, 2011, p. 101.

[4] D. Raabe, F. Roters, J. Neugebauer, I. Gutierrez-Urrutia, T. Hickel, W. Bleck, J.M. Schneider, J.E. Wittig, and J. Mayer, $A b$ initio-guided design of twinning-induced plasticity steels, MRS Bull., 41(2016), No. 4, p. 320.

[5] W. Bleck and D. Raabe, [in] The 4th International Conference on Medium and High Manganese Steels, Aachen, 2019.

[6] W. Bleck, and D. Raabe, Physical metallurgy of high manganese steels, Metals, 9(2019), No. 10, art. No. 1053.

[7] C. Alves, J. Rezende, and D. Senk, Comparison Between Segregation of high-manganese steels from 2-D phase-field simulations, 1-D analytical theories, and solidification experiments, Steel Res. Int., 87(2016), No. 9, p. 1179.

[8] S.Zaefferer and N.N.Elhami, Theory and application of electron channelling contrast imaging under controlled diffraction conditions, Acta Mater., 75(2014), p. 20.

[9] J.I. Zhang, S. Zaefferer, and D. Raabe, A study on the geometry of dislocation patterns in the surrounding of nanoindents in a TWIP steel using electron channeling contrast imaging and discrete dislocation dynamics simulations, Mater. Sci. Eng. A, 636(2015), p. 231.

[10] M. Herbig, Spatially correlated electron microscopy and atom probe tomography: Current possibilities and future perspectives, Scripta Mater., 148(2018), p. 98.

[11] M.J. Yao, E. Welsch, D. Ponge, S.M.H. Haghighat, S. Sandlöbes, P. Choi, M. Herbig, I. Bleskov, T. Hickel, M. Lipinska-Chwalek, P. Shanthraj, C. Scheu, S. Zaefferer, B. Gault, and D. Raabe, Strengthening and strain hardening mechanisms in a precipitation-hardened high-Mn lightweight steel, Acta Mater., 140(2017), p. 258.

[12] M. Herbig, P. Choi, and D. Raabe, Combining structural and chemical information at the nanometer scale by correlative transmission electron microscopy and atom probe tomography, Ultramicroscopy, 153(2015), p. 32.

[13] A. Saeed-Akbari, L. Mosecker, A. Schwedt, and W. Bleck, Characterization and prediction of flow behaviour in high-manganese twinning-induced plasticity steels: Part I. Mechanisms maps and work-hardening behavior, Metall. Mater. Trans. A, 43(2012), No. 5, p. 1688.

[14] S. Allain, J.P. Chateau, O. Bouaziz, S. Migot, and N. Guelton, Correlations between the calculated stacking fault energy and the plasticity mechanisms in Fe-Mn-C alloys, Mater. Sci. Eng. A, 387-389(2004), p. 158.

[15] P.H. Adler, G.B. Olsen, and W.S. Owen, Strain hardening of hadfield manganese steels, Metall. Mat. Trans. A, 17(1986), No. 10, p. 1725.

[16] A. Saeed-Akbari, J. Imlau, U. Prahl, and W. Bleck, Derivation and variation in composition-dependent stacking fault energy maps based on subregular solution model in high-manganese steels, Metall. Mater. Trans. A, 40(2009), No. 13, p. 3076.

[17] M. Madivala, A. Schwedt, S.L. Wong, F. Roters, U. Prahl, and W. Bleck, Temperature dependent strain hardening and fracture behavior of TWIP steel, Int. J. Plast., 104(2018), p. 80.

[18] Z.Y. Liang, Z.C. Luo, and M.X. Huang, Temperature dependence of strengthening mechanisms in a twinning-induced plasticity steel, Int. J. Plast., 116(2019), p. 192.

[19] M. Madivala, A. Schwedt, U. Prahl, and W. Bleck, Anisotropy and strain rate effects on the failure behavior of TWIP steel: A multiscale experimental study, Int. J. Plast., 115(2019), p. 178.

[20] J. von Appen, and R. Dronskowski, Carbon-induced ordering in manganese-rich austenite - A density-functional total-energy and chemical bonding study, Steel Res. Int., 82(2011), No. 2, p. 101.

[21] T.A. Timmerscheidt and R. Dronskowski, An ab initio study of carbon-induced ordering in austenitic $\mathrm{Fe}-\mathrm{Mn}-\mathrm{Al}-\mathrm{C}$ Alloys, Steel Res. Int., 88(2017), No. 1, art. No. 1600292.

[22] R.B. McLellen, Cell modells for interstitial solid solutions, Acta Metall., 30(1982), No. 1, p. 317.

[23] J.H. Kang, T. Ingendahl, J. von Appen, R. Dronskowski, and W. Bleck, Impact of short-range ordering on yield strength of high manganese austenitic steels, Mater. Sci. Eng. A, 614(2014), p. 122.

[24] J.H. Kang, T. Ingendahl, and W. Bleck, A constitutive model for the tensile behaviour of TWIP steels: Composition and temperature dependencies, Mater. Des., 90(2016), p. 340.

[25] B.C. De Cooman, Y. Estrin, and S.K. Kim, Twinning-induced plasticity (TWIP) steels, Acta Mater., 142(2018), p. 283.

[26] J.D. Yoo and K.T. Park, Microband-induced plasticity in a high Mn-Al-C light steel, Mater. Sci. Eng. A, 496(2008), No. 1-2, p. 417.

[27] S. Wesselmecking, W.W. Song, Y. Ma, T. Roesler, H. Hofmann, and W. Bleck, Strain aging behaviour of an austenitic high-Mn Steel, Steel Res. Int., 89(2018), No. 9, art. No. 1700515 .

[28] S. Sevsek, F. Brasche, C. Haase, and W. Bleck, Combined deformation twinning and short-range ordering causes serrated flow in high-manganese steels, Mater. Sci. Eng. A, 746(2019), p. 434.

[29] W.W. Song, D. Bogdanovski, A. Yildiz, J. Houston, R. Dronskowski, and $\mathrm{W}$. Bleck, On the Mn-C short-range-ordering in a 
high-strength high-ductility steel: Small angle neutron scattering and ab initio investigation, Metals, 8(2018), No. 1, art. No. 44.

[30] Y. Ma, Medium-manganese steels processed by austenite-reverted transformation annealing for automotive applications, $\mathrm{Ma}$ ter. Sci. Technol., 33(2017), No. 15, p. 1713.

[31] A. Gramlich, R. Emmrich, and W. Bleck, Austenite reversion tempering-annealing of $4 \mathrm{wt} . \%$ manganese steels for automotive forging application, Metals, 9(2019), No. 5, art. No. 575

[32] T. Allam, X.F. Guo, S. Sevsek, M. Lipinska-Chwałek, A. Hamada, E. Ahmed, and W. Bleck, Development of a $\mathrm{Cr}-\mathrm{Ni}-\mathrm{V}-\mathrm{N}$ medium manganese steel with balanced mechanical and corrosion properties, Metals, $9(2019)$, No. 6, art. No. 705.

[33] J.-K. Choi, I. W. Han, J.-T. Lee, D. Seo, and S. G. Lee, PO-
SCO High Mn Steel Debut in LNG fuelled ship and onshore LNG terminal, [in] Proceedings of the 4th International Conference on Medium and High Manganese Steels, Aachen, 2019, p. 19.

[34] H.W. Jin, N. Ma, A. Wasson, D. Fairchild, D. Lee, S. Lee, S.K. Kim, and J.K. Choi, Development of high Mn steel metallurgy for erosion resistant slurry pipeline, [in] Proceedings of the 4th International Conference on Medium and High Manganese Steels, Aachen, 2019, p. 314.

[35] P. Köhnen, S. Ewalds, J.H. Schleifenbaum, A. Belyakov, and C. Haase, Controlling microstructure and mechanical properties of additively manufactured high-strength steels by tailored solidification, Addit. Manuf., 35(2020), art. No. 101389. 